\title{
Proposta de laboratório de física de baixo custo para escolas da rede pública de ensino médio
}

\author{
Proposal for low cost physics laboratory for public high school \\ José Carlos Xavier Silva ${ }^{1}$, Carlos Eduardo dos Santos Leal ${ }^{* 2,3}$ \\ ${ }^{1}$ Instituto de Física, Universidade do Estado do Rio de Janeiro, Rio de Janeiro, RJ,Brasil \\ ${ }^{2}$ Faculdade de Engenharia, Universidade do Estado do Rio de Janeiro, Rio de Janeiro, RJ,Brasil \\ ${ }^{3}$ Engenharia, Universidade Veiga de Almeida, Rio de Janeiro, RJ,Brasil
}

Recebido em 26 de Julho, 2016. Revisado em 24 de Agosto, 2016. Aceito em 24 de Agosto, 2016

\begin{abstract}
Apresentamos neste trabalho uma proposta de construção de um laboratório de Física utilizando materiais recicláveis e de baixo custo que proporcione aos professores e alunos da rede pública de ensino médio a oportunidade de ter contato com experimentos de Física, sem que sejam necessários grandes investimentos financeiros na aquisição de equipamentos e de materiais de laboratório. Com o uso de materiais de baixo custo são propostos diversos experimentos referentes aos fenômenos físicos nas áreas da mecânica, óptica, eletricidade, magnetismo, hidrostática, termologia e física moderna que permitem de forma direta e intuitiva a verificação dos conteúdos apresentados nas aulas teóricas, assim como, o desenvolvimento de habilidades experimentais por parte dos estudantes. Além disso, espera-se que a introdução de experimentos demonstrativos também contribua como elemento fomentador da construção de um pensamento científico e o desenvolvimento da intuição física no ambiente escolar.

Palavras-chave: Laboratório de Física de Baixo Custo, Experimentos de Física no Ensino Médio, Laboratório com Materiais Recicláveis e de Baixo Custo.
\end{abstract}

We present a proposal to build a physics laboratory using recyclable materials and low-cost to provide teachers and students of the public high school the opportunity to have contact with physics experiments, without the need for large investments in the acquisition equipment and laboratory materials. With the use of low cost materials are proposed several experiments relating to physical phenomena in the fields of mechanics, optics, electricity, magnetism, hydrostatic, thermology and modern physics that allow direct and intuitive verification of the content presented in the lectures, as well as the development of experimental skills on the part of students. Moreover, it is expected that the introduction of demonstrative experiments also contribute as developer component in the construction of a scientific thinking and the development of physical intuition in the school environment.

Keywords: Physics Laboratory of Low Cost, Physics experiments in high school, Laboratory with Recyclable Materials and Low Cost.

\section{Introdução}

Observa-se no Brasil uma significativa dificuldade dos estudantes oriundos de classes sociais economicamente menos favorecidas, que normalmente se utilizam da rede pública de ensino fundamental e médio, obterem sucesso nos exames de admissão às universidades públicas. Iniciativas políticas paliativas foram introduzidas nos últimos anos, no sentido

*Endereço de correspondência: ceduardo_leal@yahoo.com.br de mitigar esse problema, ao adotar projetos facilitadores de acesso às universidades, tais como o Pró-Uni e/ou as reservas de vagas (sistema de cotas), sem, entretanto, realizar medidas efetivas para a melhoria do ensino básico. Consequentemente, as dificuldades encontradas por esses alunos persistem, pois, o problema reside fundamentalmente na péssima formação básica fornecida aos alunos da rede pública de ensino médio [1]. 
Parte dessa precariedade de formação se dá, por exemplo, devido à limitada carga horária destinada à formação das aulas teóricas de ciências; à baixa disponibilidade do uso de novas tecnologias na relação ensino-aprendizado em salas de aula; e a uma quase nula atividade experimental em laboratórios didáticos, principalmente os de Física e de Química. Esses fatores dão origem a uma formação científica e tecnológica bastante deficiente aos estudantes das escolas brasileiras da rede pública de ensino, deixando-os cada vez mais defasados em relação ao cenário de desenvolvimento tecnológico mundial.

Além disso, esses fatos corroboram, de forma bastante significativa, para o baixo número de profissionais incentivados em prosseguir carreiras de caráter científico e tecnológico e consequentemente, vir a ocupar posições de trabalho existentes nos centros de pesquisas, universidades e/ou nas indústrias. Essas dificuldades se tornam evidentes na etapa de transição entre o fim do ensino médio e a tentativa de ingresso nas grandes universidades brasileiras, por parte dos alunos oriundos da rede pública de ensino. Nessa etapa, verifica-se, em particular, que a ausência de uma boa formação na disciplina de Física é um fator determinante na triagem desses candidatos.

Ao longo dos anos dedicados ao ensino de Física temos acreditado, que grande parte da dificuldade encontrada por esses alunos quanto ao aprendizado de Física se dá pela ausência de laboratórios didáticos, que servem como instrumentos de verificação dos fenômenos físicos em complementaridade aos estudos teóricos realizados em sala de aula [2]. As atividades práticas em laboratórios fomentam nos alunos um conhecimento único a respeito da importância do aprendizado de Física e de sua aplicação no dia-a-dia, facilitando o seu aprendizado [3]. Surge, portanto, uma necessidade de aplicação de várias iniciativas de modo que as lacunas oriundas dessa má formação em Física possam ser minimizadas de maneira significativa, e que o interesse dos estudantes nas áreas de ciência e tecnologia possa ser estimulado e seus conteúdos teóricos e práticos sejam assimilados com maior clareza.

Neste sentido, desenvolvemos neste trabalho uma proposta de construção de laboratórios didáticos de Física voltados para atender as necessidades dos conteúdos das três séries do nível médio da rede pública de ensino. A elaboração de um conjunto de equipamentos de simples confecção e a realização de experimentos para a verificação de diversos fenômenos físicos utilizando materiais recicláveis e de baixo custo tem apresentado resultados muito interessantes em termos de ensino de Física [4].

Cabe ressaltar que todo o material que compõe os kits de experimentos do laboratório é de fácil aquisição, baixos custos e de grande facilidade de manuseio, podendo ser produzido por professores e pelos próprios alunos, o que contribui sobremaneira para o desenvolvimento de habilidades experimentais dos participantes e de sua implantação em escolas públicas que dispõem de poucos recursos financeiros para montagem de laboratórios didáticos. Com uma estrutura básica o laboratório didático de Física de baixo custo atende integralmente aos conteúdos das três series do ensino médio e permite aos estudantes desenvolver experimentos quantitativos nas áreas de Mecânica (estática e dinâmica), Eletricidade e Magnetismo, Óptica, Hidrostática, Termologia e até de Física Moderna.

Por fim, a montagem de atividades de laboratório de caráter meramente qualitativa pode ser utilizada de forma complementar em sala de aula e também em apresentações em Feiras de Ciências, como forma de validar a ocorrência dos fenômenos físicos no diaa-dia e também, para fortalecer a intuição física no ambiente escolar a partir do exercício do pensamento científico.

\section{Metodologia}

A proposta de implementação de um laboratório didático de Física utilizando materiais de baixo custo em escolas públicas do ensino médio visa prestar um serviço direto à escola, aos seus professores e alunos ao procurar suprimir deficiências dessas escolas em termos de atividades práticas de laboratório, proporcionando aos estudantes um ensino de melhor qualidade nas aulas de Física. Outro aspecto relevante dessa proposta é auxiliar na capacitação e aprimoramento dos professores de Física dessas instituições de ensino quanto à construção de equipamentos de baixos custos e a realização de experimentos simples voltados para a realização de práticas de laboratório e apoio às aulas teóricas. Além disso, de forma indireta o projeto agrega valor a toda sociedade por contribuir para melhoria da formação científica das novas gerações. 
A proposta se inicia a partir da execução de um cronograma de atividades no qual são definidas as experimentações a serem realizadas para as diferentes séries da escola de ensino médio. Em seguida, é realizado um levantamento bibliográfico para a confecção de roteiros dos experimentos selecionados. A etapa seguinte consiste da tomada de preço e aquisição de materiais necessários para a construção dos equipamentos referentes ao conjunto de experimentos para cada uma das três séries, a saber: $1^{a}$ série - Termologia e Óptica, $2^{a}$ série - Mecânica (estática, dinâmica e hidrostática), e $3^{a}$ série (Eletricidade e Magnetismo e Física Moderna). Com as experiências e roteiros prontos e testados, são iniciadas as práticas referentes às experiências de laboratório de Física correspondentes a cada série escolar. Por fim, é realizada a coleta de dados e a confecção de relatórios, pelos alunos, para os experimentos realizados.

\section{Resultados - Elaboração de Experimentos}

Nesta seção, apresentamos um conjunto básico de experimentos factíveis de realização num laboratório didático de Física para uma escola de ensino médio, sem a necessidade de grandes investimentos financeiros por utilizar materiais de baixo custo, tais como, tubos de PVC, garrafas PET, fios, parafusos, réguas, dinamômetros, apontador laser, entre outros.

A construção de equipamentos para realização de experimentos e roteirização dos conteúdos de práticas de Mecânica (estática e dinâmica) tem como base a construção de um "trilho de ar", (vide Figura 1), utilizando tubos e conexões de PVC e diversos

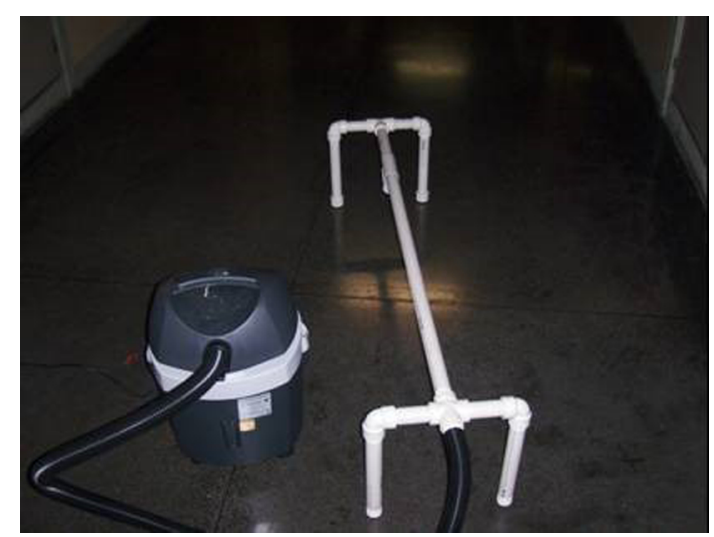

Figura 1: Trilho de ar construído com tubos e conexões de PVC e um compressor de ar orifícios de diâmetro de décimos de milímetro ligados a um compressor de ar, que permite aos professores e alunos a realização de diversos experimentos relacionados ao estudo dos movimentos retilíneo uniforme e uniformemente variado, determinação da aceleração da gravidade e colisões entre outros $[5,6]$.

A fácil construção do equipamento "trilho óptico ou banco óptico", (vide Figura 2), utiliza conexões tipo rosca (1/2, 3/4 e 1 polegada) e tubos de PVC (1,5 m de extensão e $3 / 4$ de polegada) tendo bases de apoio e hastes móveis [7]. Este aparato permite a realização de diversas práticas de laboratório de óptica geométrica, tais como: propagação retilínea da luz, formação de imagens em espelhos planos, côncavos e convexos, câmara escura, lei de Snell, entre outras.

As atividades de laboratório de Termologia são baseadas na construção de equipamentos simples e de fácil manuseio tais como calorímetros de baixo custo e coletor solar térmico para aquecimento de água utilizando garrafas PET e caixas Tetrapak [8]. Além disso, são propostas experiências para verificação da mudança de fase das substâncias, equilíbrio térmico, $1^{a}$ e $2^{a}$ leis da Termodinâmica, transferência de calor por condução, relação pressão e temperatura, entre outros.

O módulo básico referente às atividades de laboratório de Eletricidade e Magnetismo é construído a partir do uso de vários equipamentos de fácil manuseio, como por exemplo: eletroscópios com tubo de papelão e folha de alumínio; o uso de materiais simples como canudos plásticos, ímãs e resistores; e a aquisição de equipamentos comerciais, tais como: transformadores e multímetros. Com essas ferramentas são elaboradas atividades práticas para a

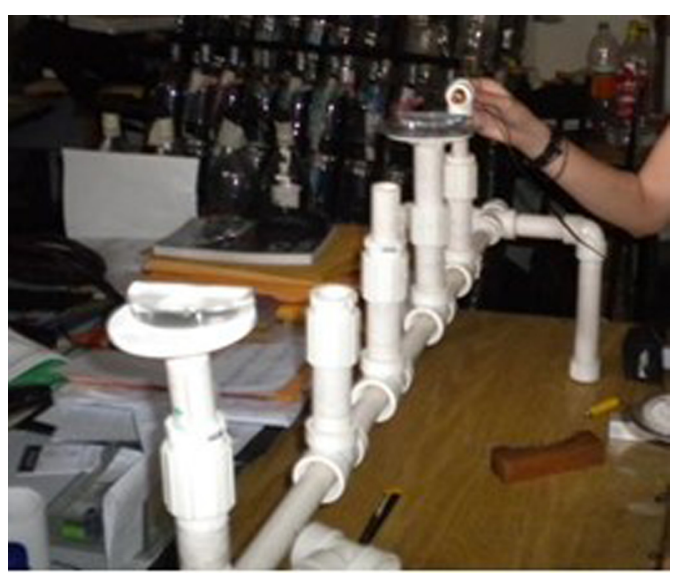

Figura 2: Trilho óptico com tubos e conexões de PVC 
verificação das leis e fenômenos fundamentais da eletricidade e do magnetismo: eletrização dos corpos; lei de Coulomb; lei de Gauss em corpos metálicos; lei de Ohm; lei de indução de Faraday; leis de Kirchhoff em circuitos elétricos; lei de atração e repulsão magnética; associação de resistores em série e em paralelo, entre outras.

\section{Estudo de caso}

No período 2007-2008 foi implementada a proposta Laboratório Didático de Física de Baixo Custo numa escola da rede pública de ensino médio no Rio de Janeiro. O projeto foi realizado a partir de recursos da FAPERJ, segundo o programa de Apoio à Melhoria do Ensino nas Escolas Públicas/RJ, 200\%. A unidade escolar atendida foi o Colégio Estadual João Alfredo - CEJA, que apresentava uma grande carência de equipamentos e atividades no laboratório didático de ciências.

O projeto contou com a participação efetiva de dois professores de Física do Colégio João Alfredo, como bolsistas da FAPERJ, e de um grupo de estudantes do curso de licenciatura em Física-UERJ, que participaram com bolsas de Iniciação à Docência. Esses estudantes atuaram na construção dos equipamentos, na confecção dos experimentos e dos roteiros das experiências, assim como, no auxílio aos professores durante as aulas práticas de laboratório.

Essa integração Universidade-Escola contribuiu de forma bastante significativa para estimular o desenvolvimento de habilidades experimentais nos futuros licenciados de Física e na melhoria da formação experimental dos professores da escola de ensino médio atendida pelo projeto. Além disso, foram observadas significativas melhorias no aproveitamento dos alunos do nível médio na relação teoria-experimentação durante as aulas de Física.

A proposta de implementação do laboratório de Física no Colégio Estadual João Alfredo atendeu a cerca de mil alunos, distribuídos nas diversas turmas/turnos das três series, conforme Quadro I.

No intuito de fortalecer a construção de um pensamento científico e o desenvolvimento da intuição física naquele ambiente escolar foram realizadas diversas outras atividades práticas qualitativas, com materiais simples e de baixo custo, para fins de utilização e apoio em salas de aula, como também em apresentações em Feiras de Ciências, a saber: Figuras de Lissajous, Torre de Pisa, Pêndulos Ressonantes, Relógio Solar, Giroscópio, Efeito Fotoelétrico etc.

Os indicadores extraídos desse trabalho de pesquisa estão ligados ao expressivo número de alunos das três séries da escola de ensino médio que foram beneficiados com o projeto. Segundo os questionários de avaliação aplicados, os relatórios elaborados e a opinião dos professores envolvidos foram percebidos uma melhoria de desempenho nas aulas teóricas da disciplina de Física estimulados pela verificação prática dos fenômenos físicos no laboratório, estabelecendo um ganho significativo na relação ensinoaprendizado.

\section{Conclusão}

A implementação de laboratórios didáticos de Física, tendo como base a construção de equipamentos e a realização de um conjunto de experimentos qualitativos e quantitativos, utilizando materiais recicláveis e/ou de baixo custo pode se tornar uma excelente oportunidade pedagógica e de grande incentivo ao desenvolvimento científico nas escolas públicas de ensino médio. Além disso, o trabalho visa à melhoria da participação e do aprendizado dos alunos, nas aulas teóricas de Física, estimulado pela verificação dos fenômenos físicos nas atividades de laboratório, melhoria da capacitação dos professores responsáveis pela disciplina de Física, no que concerne a formação em Física Experimental e a preparação de experimentos simples e de baixos custos para a preparação das aulas práticas.

Apresentamos uma breve descrição de um estudo de caso de implementação de um laboratório de Física, de baixo custo, no Colégio Estadual João Alfredo, no Rio de Janeiro. Esta iniciativa possibilitou uma forte integração entre docentes e estudantes universitários da UERJ com os professores e alunos da referida escola. Além disso, cerca de 1.000 (mil)

Quadro 1: Relação turmas atendidas no laboratório de Física

\begin{tabular}{lccc}
\hline Número de turmas por turno & $1^{a}$ serie & $2^{a}$ serie & $3^{a}$ serie \\
\hline (laboratório de Física) & (calor e óptica) & (mecânica) & (eletricidade e magnetismo) \\
\hline Turmas/manhã & 03 & 03 & 01 \\
Turmas/tarde & 13 & 06 & - \\
\hline
\end{tabular}


alunos das três séries escolares tiveram a oportunidade de desenvolver habilidades na confecção de equipamentos científicos e de verificar a ocorrência de fenômenos físicos durante a realização das atividades experimentais, o que contribuiu significativamente para a melhoria do nível de conhecimentos científicos desses alunos nas áreas de mecânica, termologia, eletricidade, magnetismo, óptica e física moderna.

Por fim, temos em mente que inciativas voltadas para a promoção e estímulo do desenvolvimento científico nas escolas públicas, tais como a implementação de laboratórios didáticos de baixo custo em Ciências (Física, Química e Biologia), devam ser incentivadas e apoiadas pelos órgãos de fomento e sejam tratadas como políticas educacionais junto à rede pública de ensino. De outra forma, estaremos condenando nossos alunos, em pleno século XXI, a viverem excluídos do conhecimento científico que precede as grandes descobertas e invenções tecnológicas e a assistirem a chegada do futuro e o desenvolvimento tecnológico mundial na condição de meros expectadores.

\section{Agradecimentos}

Os autores agradecem a FAPERJ (Processo $n^{\circ}$ E26/110.222/2007) por ocasião do apoio financeiro para realização dessa proposta no Colégio Estadual João Alfredo (CEJA) no Rio de Janeiro, e aos professores do CEJA e alunos do Instituto de Física da UERJ que participaram de sua implementação.

\section{Referências}

[1] J.C. Xavier, in XV Simpósio Nacional de Ensino de Física, Curitiba, 2003, disponível em http://www.sbf1.sbfisica.org.br/eventos/ snef/xv/trabalhos/trabupload/R138421.pdf.

[2] W.C.S. Eiras, in IV Encontro Nacional de Pesquisa em Educação em Ciências, Bauru, 2002, disponível em http://fep.if.usp.br/ profis/arquivos/ ivenpec/Arquivos/Orais/ORAL093.pdf

[3] M.G. Séré, S.M. Coelho, A.D. Nunes, Caderno Brasileiro de Ensino de Física 20, 30 (2003).

[4] J.C. Xavier, C.E.S. Leal e L.P. Brandão, in Anais do $4^{\circ}$ Congresso Brasileiro de Extensão Universitária, Dourados, 2009, disponível em http://portal.ufgd.edu.br/arquivos/ apresentacoes_posteres.pdf.

[5] J.C.X. Silva, C.E.S. Leal, L.F. Barbosa, L.F. Santos, M.B. Correa, P.R. Pessanha, S.R. Azeredo,
A. Alves, L.P. Brandão, S.M. Lanes, T. Fejolo e W.J. Silva, in XVIII Simpósio Nacional de Ensino de Fúsica, Vitória, 2009, disponível em http://www.cienciamao.usp.br/dados/snef/ trilhodeardepvcparaoestu.trabalho.pdf.

[6] L.F. Barbosa, J.C.X. Silva, C.E.S. Leal, A. Alves, M.B. Correa e N.S. Dias, in 62 Reunião Anual da SBPC, Natal, 2010, disponível em http://www.sbpcnet.org.br/livro/ 62ra/resumos/resumos/973.htm

[7] A.A. Oliveira Júnior, J.C.X. Silva, C.E.S. Leal, M.B. Correa, L.F. Barbosa e N.S. Dias, in 62 ${ }^{a}$ Reunião Anual da SBPC, Natal, 2010, disponível em http://www.sbpcnet.org.br/livro/ 62ra/resumos/resumos/1059.htm.

[8] J.A. Alano, Aquecedor Solar Composto de Produtos Descartáveis. Manual de Construção e Instalação (Celesc Distribuição S.A., Florianópolis, 2009), disponível em http://novoportal.celesc.com br/portal/images/arquivos/manuais/manualaquecedor-solar.pdf. 